\title{
Polarimetry with the Gemini Planet Imager: Instrument Characterization and Future Science
}

\author{
Max Millar-Blanchaer ${ }^{1}$, Sloane J. Wiktorowicz ${ }^{2}$, Marshall D. \\ Perrin $^{3}$, James R. Graham ${ }^{4}$, Sandrine J. Thomas ${ }^{5}$, Daren Dillon ${ }^{6}$, \\ Michael P. Fitzgerald ${ }^{7}$, Jérome Maire ${ }^{8}$, Bruce Macintosh ${ }^{9}$ and \\ Stephen J. Goodsell ${ }^{10}$ \\ ${ }^{1}$ Dept of Astronomy and Astrophysics, University of Toronto, ON, M5S 3H4, Canada \\ email: maxmb@astro.utoronto.ca \\ ${ }^{2}$ Astronomy and Astrophysics Dept., University of California at Santa Cruz, CA, 95064, USA \\ ${ }^{3}$ Space Telescope Science Institute, Baltimore, MD, 21218, USA \\ ${ }^{4}$ Astronomy Department, University California at Berkeley, CA, 94720, USA \\ ${ }^{5}$ NASA Ames Research Center, Moffett Field, CA 94035, United States \\ ${ }^{6}$ UC Observatories, University of California, Santa Cruz, CA, 95064, USA \\ ${ }^{7}$ Department of Physics \& Astronomy, University of California, Los Angeles, CA, 90095, USA \\ ${ }^{8}$ Dunlap Institute for Astronomy \& Astrophysics, University of Toronto, ON, M5S 3H4, \\ Canada \\ ${ }^{9}$ Lawrence Livermore National Laboratory, CA, 94551, USA \\ ${ }^{10}$ Gemini Observatory, Hilo, Hawaii, 96720, USA
}

\begin{abstract}
The Gemini Planet Imager (GPI) is a high contrast coronagraph designed to directly image exoplanets and circumstellar disks. GPI includes a polarimetry mode designed to characterize dust grains and enhance the contrast of scattered, polarized light by a factor of 100 . Reflections and birefringence of optics within the optical train induce a polarization signature that needs to be measured a priori and calibrated out during data reduction. Here we report on the results of an extensive laboratory characterization campaign of the polarimetry mode. The linear instrumental polarization has been measured in 4 GPI passbands and found to be between $3.5 \pm 0.3 \%$ at 1.0 micron and $1.1 \pm 0.3 \%$ at 2.0 microns. Modulation efficiency has been measured to be $94 \%$ at 1.0 micron increasing to $97 \%$ at 2.0 microns. Stability has been shown to better than $0.6 \%$ over timescales of $\sim 3$ months and over cool down cycles. The tests show that GPI passes all polarimetry design requirements and should be able to measure circumstellar disk linear polarization to $1 \%$ accuracy.
\end{abstract}

\section{Disk Science with GPI}

The study of debris disks has been shown to be an invaluable tool in the characterization of planetary systems. Disk morphology has been used to predict the presence of unseen companions - e.g. Kalas et al. 2005, Lagrange et al. 2009 and Marois et al. 2008 - and provide constraints on planetary masses and orbital elements. In addition, the polarization signature of disks can provide information on the size distribution of dust grains (e.g. Graham et al. 2007). The GPI Exoplanet Survey (GPIES) campaign has been granted a total of 890 hours of observing time, 35 of which has been earmarked for debris disk polarimetry: 10 hours for snapshots of stars predicted to have detectable debris disks and 25 hours for high SNR images of disks with apparent planet-induced structure. GPIs polarimetry mode is implemented via a Wollaston prism, a half wave plate, and software for reconstructing Stokes parameter datacubes (Perrin et al. 2010 and Maire et al. 2010). 
Table 1. The instrumental polarization is stable to $0.6 \%$. over 3 month timescales

\begin{tabular}{ccc}
\hline GPI Passband & Instrumental Linear Polarization & Instrumental Phase Retardance \\
\hline Y & $3.54 \pm 0.35 \%$ & $-10.6 \pm 1.1^{\circ}$ \\
J & $3.00 \pm 0.50 \%$ & $-5.2 \pm 0.50^{\circ}$ \\
H & $2.37 \pm 0.28 \%$ & $-3.58 \pm 0.95^{\circ}$ \\
K1 & $1.14 \pm 0.33 \%$ & $-2.34 \pm 0.60^{\circ}$ \\
\hline
\end{tabular}
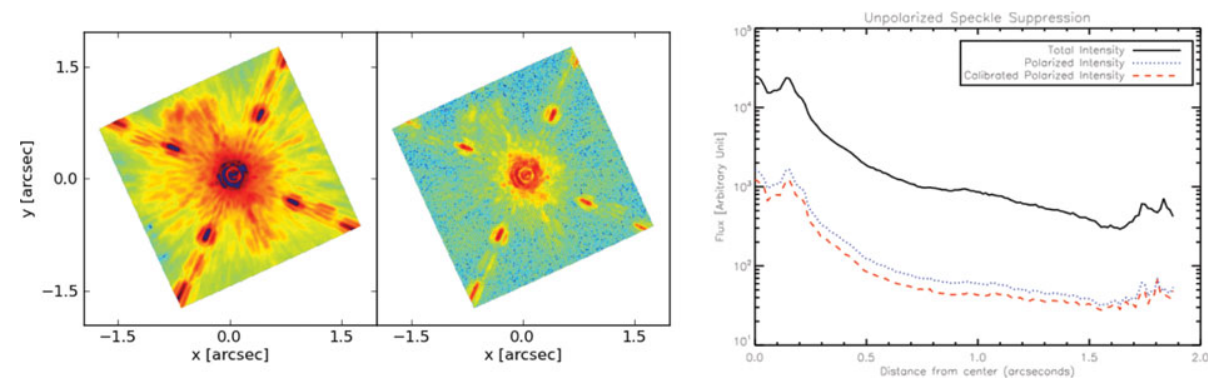

Figure 1. Polarimetry mode total intensity (left) and linearly polarized intensity (center). Intensity as a function of position from the central spot (right).

\section{Instrumental Polarization}

Calibration of the subsystem was carried out at UC Santa Cruz. The instrumental polarization has been measured and we have reached our performance goals in all cases except the $\mathrm{Y}$ band phase retardance, see Table 1 . The measurements indicate that we should be able to achieve on-sky accuracy of $\sim 1 \%$ (Perrin et al. 2010).

\section{Speckle Suppression}

In a set of coronagraphic images the intensity within $1^{\prime \prime}$ of the central spot was reduced by a factor of 20 when using linearly polarized intensity opposed to total intensity, see Figure 1. Performance here is limited by the polarization of the laboratory light source $(\sim 10 \%)$ and high read noise, so these values should be taken as a lower limit on expected performance.

\section{Conclusions}

The GPI Polarimetry subsystem has met all laboratory performance requirements and is expected to achieve an on-sky accuracy of $1 \%$ when measuring linear polarization. These results have been replicated over timescales of 3 months. The use of linear polarized light has been shown to provide an effective means of suppressing residual speckles.

\section{References}

Graham, J. R., Kalas, P. G., \& Matthews, B. C. 2007, ApJ, 654, 595

Kalas, P., Graham, J. R., \& Clampin, M. 2005, Nature, 435, 1067

Lagrange, A.-M., Gratadour, D., Chauvin, G., et al. 2009, A\&A, 493, L21

Macintosh, B. A., Graham, J. R., Palmer, D. W., et al. 2008, SPIE, 7014, 31

Maire, J., Perrin, M. D., Doyon, R., et al. 2010, SPIE, 7735, 102

Marois, C., Macintosh, B., Barman, T., et al. 2008, Science, 322, 1348

Perrin, M. D., Graham, J. R., Larkin, J. E., et al. 2010, SPIE, 7736, 192 